\title{
UNIVERSAL QUANTUM COMPUTATION WITH METAPLECTIC ANYONS
}

\author{
SHAWN X. CUI ${ }^{1}$ AND ZHENGHAN WANG ${ }^{1,2}$
}

\begin{abstract}
We show that braidings of the metaplectic anyons $X_{\epsilon}$ in $S O(3)_{2}=S U(2)_{4}$ with their total charge equal to the metaplectic mode $Y$ supplemented with projective measurements of the total charge of two metaplectic anyons are universal for quantum computation. We conjecture that similar universal anyonic computing models can be constructed for all metaplectic anyon systems $S O(p)_{2}$ for any odd prime $p \geq 5$. In order to prove universality, we find new conceptually appealing universal gate sets for qutrits and qupits.
\end{abstract}

\section{INTRODUCTION}

Anyons are modeled mathematically by simple objects of unitary modular categories (UMCs). An important invariant of an anyon type $x$ is its quantum dimension $d_{x}$-the ground state degeneracy $V_{n, x}$ of $n$ type $x$ anyons in the disk $D^{2}$ (with an appropriate total charge) is asymptotically $d_{x}^{n}$. When $d_{x}=1$, an anyon of type $x$ is abelian. Otherwise, $d_{x}>1$ and such anyons are non-abelian. A non-abelian anyon of type $x$ naturally leads to a representation $\rho_{n, x}$ of the $n$-stand braid group $B_{n}: B_{n} \rightarrow U\left(V_{n, x}\right)$ for each $n \geq 1$. The property $F$ conjecture is that the images $\rho_{n, x}\left(B_{n}\right)$ in $U\left(V_{n, x}\right)$ are all finite subgroups if and only if $d_{x}^{2} \in \mathbb{Z}[19]$. When $d_{x}^{2} \in \mathbb{Z}$, then anyons of type $x$ are called weakly integral. Interesting weakly integral anyons include those in metaplectic UMCs [13], which are known to have Property $F$ [23].

Anyons can be used for quantum information processing. Ideally, we would like to have a non-abelian anyon such as the Fibonacci anyon whose braidings alone are universal for quantum computation [10]. But more realistic anyons seem to be weakly integral. If the Property $F$ conjecture holds, then weakly integral anyons cannot be universal for quantum computation by braidings alone. Therefore, it is interesting

Date: Revised Feb. 26th 2015.

Key words and phrases. anyonic quantum computation, metaplectic anyons, universal gate set, braid group.

The authors are partially supported by NSF DMS 1108736 . 
to investigate what extra resources are required for universal quantum computation. In [8], we analyze the simplest integral non-abelian UMC $D\left(S_{3}\right)$. In this paper, we focus on anyons in the metaplectic UMCs. We separate weakly integral anyons into two classes [2]: $P$-anyons and $\# P$-anyons. $P$-anyons are those whose associated link invariants can be computed classically in polynomial time, while the associated link invariants of $\# P$-anyons are $\# P$ hard to compute. In particular all abelian anyons are $P$-anyons. Abelian anyons are only good for topological quantum memory because the resulting braid group representations only lead to phases[1. While the Ising anyon leads to many topologically protected quantum gates, all can be simulated classically efficiently because they are Clifford gates [20. Moreover, we believe the projective measurements of the total charge of any number of Ising anyons can also be simulated classically efficiently. The Ising anyon and the metaplectic anyon $X_{\epsilon}$ of quantum dimension $=\sqrt{p}$ in $S O(p)_{2}$ are all $P$-anyons. Surprisingly, the metaplectic modes $Y_{i}$ of quantum dimension $=2$ in the metaplectic UMCs are $\# P$-anyons [13]. This $\# P$-hardness makes us believe that if the metaplecitc modes $Y_{i}$ 's are used in the computation, we might gain extra computational power. Indeed, we will show that braidings of the metaplectic anyons $X_{\epsilon}$ in $S O(3)_{2}=S U(2)_{4}$ with their total charge equal to the metaplectic mode $Y$ supplemented with projective measurements of the total charge of two metaplectic anyons are universal for quantum computation. We conjecture that similar universal computing models can be constructed for all metaplectic anyon systems $S O(p)_{2}$ for any odd prime $p \geq 5$. In order to prove universality, we find new conceptually appealing universal gate sets for qutrits and qupits.

Our interest for $S U(2)_{4}$ comes from its potential physical relevance. There are many possible routes to realize our universal quantum computational model: fractional quantum Hall liquids at $\nu=8 / 3$ [22], bilayer fractional quantum Hall liquids at $\nu=2 / 3$ [4], metaplectic anyons [12, and parafermion zero modes [7]. Evidence for the realization of $S U(2)_{4}$ in fractional quantum Hall liquids at $\nu=8 / 3$ is found numerically [21]. The experimental challenge is to find a realization of the metaplectic mode $Y$ of dimension $=2$.

The paper is organized as follows. In Section 2, we give two new universal gate sets, one for qutrit and one for qupit. In Section 3 , we present a universal anyonic model with $S U(2)_{4}$ and use theorems in Section 2 to prove its universality. We also propose a similar model with $S O(5)_{2}$ and provide some partial results. Appendices $\mathrm{A}$ contain the $6 j$-symbols and $R$-symbols for $S O(3)_{2}$ and $S O(5)_{2}$, which are the data we need to compute the braid group representations and construct 
braiding quantum gates. Appendix $\mathrm{C}$ shows how to compute the braid matrices for 1-qudit models.

\section{UNIVERSAL GATE SETS FOR QUTRITS AND QUPITS}

Throughout this paper, $d \geq 2$ is an integer and $\omega_{d}=e^{\frac{2 \pi i}{d}}$ is the $d$-th root of unity. We will set $\omega=\omega_{3}$, and use $p$ to denote an odd prime $p \geq 5$.

Let $\mathbb{C}^{d}$ be the qudit with the standard basis $\{|j\rangle \mid j=0,1, \ldots, d-1\}$. For $p$ an odd prime $p \geq 5$, we will refer to a qudit as a qupit. It is not hard to believe that qubits and qutrits behave differently from qupits. Our universal gate sets below show some differences already.

A standard universal gate set for the qubit quantum circuit model consists of the Hadamard gate $H$, the controlled-NOT gate CNOT, and the $\frac{\pi}{8}$-gate $T$ [5] [20]. There are natural generalizations of the Hadamard and CNOT gates to qudits. The $T$ gate is a 4 -th root of the Pauli $\sigma_{z}$ matrix. If we propose generalizations of the Pauli $\sigma_{z}$ to qudits, how many roots do we need to take for obtaining a universal gate set? For our generalizations of the Pauli matrix, the answer is simply 2 for qutrits and none for qupits.

The generalized Hadamard gate for qudits is the generalized Hadamard gate $H_{d}$ :

$$
H_{d}|j\rangle=\frac{1}{\sqrt{d}} \sum_{i=0}^{d-1} \omega_{d}^{i j}|i\rangle, j=0,1, \cdots, d-1 .
$$

A natural generalization of the CNOT gate is the following SUM gate:

$$
\mathrm{SUM}_{d}|i, j\rangle=|i, i+j(\bmod d)\rangle, i, j=0,1, \cdots, d-1 .
$$

The $T$-gate is the 4 -th root of the Pauli $\sigma_{z}$ matrix. The $\sigma_{z}$ gate can be generalized to the $Q[i]$ gates for qudits:

$$
Q[i]_{d}|j\rangle=\omega_{d}^{\delta_{i j}}|j\rangle, i, j=0,1, \cdots, d-1 .
$$

Related to the $Q[i]$ gates are the $P[i]$ gates:

$$
P[i]_{d}|j\rangle=\left(-\omega_{d}^{2}\right)^{\delta_{i j}}|j\rangle, i, j=0,1, \cdots, d-1 .
$$

Some other gates that will be used throughout this paper are:

The generalized $X$ gate, $X_{d}|i\rangle=|i+1(\bmod d)\rangle$,

The generalized $Z$ gate, $Z_{d}|i\rangle=\omega_{d}^{i}|i\rangle$,

The generalized controlled- $Z$ gate, $\bigwedge(Z)_{d}|i, j\rangle=\omega_{d}^{i j}|i, j\rangle$,

Sign-flip gate, Flip $[i]_{d}|j\rangle=(-1)^{\delta_{i j}}|j\rangle, i=0,1, \cdots, d-1$. 
When $d=3$, the $P[i]$ gate is a square root of the $Q[i]$ gate. In general when $p$ is an odd prime, $Q[i]_{p}$ is always a power of $P[i]_{p}$. When no confusion arises, we will drop the subscripts $d$ or $p$ from the notation.

We will prove below that for $d=3$ the gate set consisting of the generalized Hadamard gate $H_{3}$, the SUM gate $\mathrm{SUM}_{3}$, and any one of the $P[i]_{3}, i=0,1,2$ gates is universal for the qutrit quantum circuit model, while for qupits the generalized Hadamard gate $H_{p}$, the SUM gate $\mathrm{SUM}_{p}$, and the $Q[i]_{p}, i=1,2, \ldots, p-1$ gates suffice. Our universal qutrit gate set is new. The universal qupit gate set is distilled from the universal gate sets in 3], though our universal gate set is not explicitly given there and our proof of universality is new.

\subsection{Universal qutrit gate sets.}

Theorem 1. The following gate set is universal for the qutrit quantum circuit model:

1). The generalized Hadamard gate $H_{3}$

2). The SUM gate $\mathrm{SUM}_{3}$

3). Any gate from the set $\left\{P[0]_{3}, P[1]_{3}, P[2]_{3}\right\}$.

Remark 1. The universal set above has a strong analogy with the standard qubit universal set $\{\mathrm{CNOT}, H, T=\pi / 8$-gate $\}$ in that $\left\{\mathrm{SUM}_{3}, H_{3}, P[2]_{3}^{2}\right\}$ generate the qutrit Clifford group while $\left\{\mathrm{CNOT}, H, T^{2}\right\}$ generate the qubit Clifford group. In this sense, our universal qutrit gate set above is a natural generalization of the standard universal qubit set.

To prove the theorem, we need the following lemmas.

Lemma 1. [16] Let $U_{1}, U_{2}$ be two non-commuting matrices in $S U(2)$. If they are both of infinite order, then the subgroup generated by $U_{1}, U_{2}$ is dense in $S U(2)$.

Lemma 2. [16] Let $V$ be any finite dimensional Hilbert space. Let $H \subset S U(V)$ be the stabilizer of some non-zero vector $|\psi\rangle \in V$ and $U \in$ $S U(V)$ be any operator which does not preserve the space $\operatorname{span}\{|\psi\rangle\}$, then the set of operators $\left\{H \bigcup U^{-1} H U\right\}$ generate a dense subgroup of $S U(V)$.

Definition 1. 6] 1). A vector $|\psi\rangle \in \mathbb{C}^{d} \otimes \mathbb{C}^{d}$ is called decomposable if $|\psi\rangle=\left|\psi_{1}\right\rangle \otimes\left|\psi_{2}\right\rangle$ for some $\left|\psi_{1}\right\rangle,\left|\psi_{2}\right\rangle \in \mathbb{C}^{d}$.

$2)$. A quantum gate $U \in \mathrm{U}\left(\mathbb{C}^{d} \otimes \mathbb{C}^{d}\right)$ is primitive if it maps decomposable states to decomposable states. Otherwise, $U$ is called imprimitive.

Lemma 3. The gate $\mathrm{SUM}_{d}$ is imprimitive. 
Proof Consider the decomposable state $\frac{1}{\sqrt{d}} \sum_{i=0}^{d-1}|i\rangle \otimes|0\rangle$. It is mapped, by $\mathrm{SUM}_{d}$, to $\sum_{i=0}^{d-1} \frac{1}{\sqrt{d}}|i\rangle \otimes|i\rangle$, which is not a decomposable state.

Set $W[i]=H_{3} P[i]_{3} H_{3}^{-1} P[i]_{3}^{-1}, Z[i]=H_{3} P[i]_{3}^{-1} H_{3}^{-1} P[i]_{3}, i=0,1,2$.

Lemma 4. The generalized Hadamard $H_{3}$ and any gate from $\left\{P[0]_{3}, P[1]_{3}, P[2]_{3}\right\}$ generate a dense subgroup of $S U(3)$.

Proof Direct calculations show that $W[i]$ and $Z[i]$ both have eigenvalues $\left\{\frac{2 \pm i \sqrt{5}}{3}, 1\right\}$. Moreover, $W[i]$ and $Z[i]$ share an eigenvector with eigenvalue 1 , which is the following vector $E_{i}$, respectively, for $i=$ $0,1,2$,

$E_{1}=-|1\rangle+|2\rangle, \quad E_{2}=-\omega|0\rangle+|2\rangle, \quad E_{3}=-\omega|0\rangle+|1\rangle$.

Clearly, $\frac{2 \pm i \sqrt{5}}{3}$ are the roots of the irreducible polynomial $3 x^{2}-4 x+3$, which is not a cyclotomic polynomial. Thus $\frac{2 \pm i \sqrt{5}}{3}$ are not roots of unity. Restricted to $E_{i}^{\perp}$, the two dimensional orthogonal complement of $E_{i}$, $W[i]$ and $Z[i]$ are of infinite order. It is straightforward to check that $W[i]$ and $Z[i]$ do not commute. By Lemma $1, W[i]$ and $Z[i]$ generate a dense subgroup of $S U\left(E_{i}^{\perp}\right)$.

Since $H_{3}$ does not preserve $\operatorname{span}\left\{E_{i}\right\}$, it follows from Lemma 2 that $S U\left(E_{i}^{\perp}\right) \bigcup H_{3}^{-1} S U\left(E_{i}^{\perp}\right) H_{3}$ generate a dense subgroup of $S U(3)$. Therefore, $\left\{H_{3}, P[i]_{3}\right\}$ generate a dense subgroup of $S U(3)$.

Proof of Theorem 1 By Theorem 1.3 in [6], the collection of 1qudit gates with any imprimitive 2-qudit gate form a universal gate set for $d \geq 3$. By Lemma 3, $\mathrm{SUM}_{3}$ is an imprimitive 2-qutrit gate. By Lemma $4, H_{3}$ and any gate from $\left\{P[0]_{3}, P[1]_{3}, P[2]_{3}\right\}$ generate a dense subgroup of the group of all 1-qutrit gates. Thus, the gates from our theorem form a universal gate set.

To state the next theorem, we introduce a qutrit coherent projective measurement.

Measurement 1 . The projection of a state in the qutrit space $\mathbb{C}^{3}$ to $\operatorname{span}\{|0\rangle\}$ and its orthogonal complement $\operatorname{span}\{|1\rangle,|2\rangle\}$ so that the resulting state, if it is in $\operatorname{span}\{|1\rangle,|2\rangle\}$, is coherent.

Theorem 2. The following gate set is universal for the qutrit quantum circuit model.

1). The generalized Hadamard gate $H_{3}$

2). The SUM gate $\mathrm{SUM}_{3}$

3). Any gate from $\left\{Q[i]_{3}, i=0,1,2\right\}$ 
4). Any non-trivial 1-qutrit classical gate not equal to $H_{3}^{2}$.

5). Measurement 1

Remark 2. (1) In [8], a stronger theorem is proved: the gate set in Theorem 2 with the gate from 3 ) removed is already universal. We proved this stronger theorem by picking a qubit $\mathbb{C}^{2}$ inside a qutrit $\mathbb{C}^{3}$ and showing that one can approximate arbitrary unitary $U \in S U\left(2^{n}\right)$. We can then deduce universality for the qutrit circuit by encoding a qutrit with two qubits $\mathbb{C}^{2} \otimes \mathbb{C}^{2} \subset \mathbb{C}^{3} \otimes \mathbb{C}^{3}$. For instance, we can use $|00\rangle,|01\rangle,|10\rangle$ to encode $|0\rangle,|1\rangle,|2\rangle$, respectively. And the basis element $|11\rangle$ is left unused. But it is not known if the reduced qutrit set can be used to approximate arbitrary qutrit gates directly (i.e. not by encoding a qutrit with two qubits). Neither is it known if the gates from 3 can be constructed out of the reduced gate set.

(2) Comparing this theorem with Corollary 1 below, we see another difference between qutrit and qupits: the analogous gates through 1) to 4) are already a universal gate set for the qupit quantum circuit model, but not so for the qutrit model.

(3) If we restrict the choice of gate from 3) on $i=1,2$, then we can drop the gate in 4) while still keep the universality of the rest. This is because $H_{3}^{2}$ is the classical gate which swaps $|1\rangle$ and $|2\rangle$, so with $H_{3}$ and one of $Q[1]_{3}, Q[2]_{3}$, we can obtain the other one. Since $Z_{3}=Q[1]_{3} Q[2]_{3}^{2}$, and $X_{3}=H_{3}^{-1} Z_{3} H_{3}$, we can construct the generalized $X$ gate $X_{3}$, which is a classical gate not equal to $\mathrm{H}_{3}$.

Proof We prove this theorem by showing that we can construct all the gates in Theorem 1. Since $H_{3}^{2}$ is a classical gate, the gate from 4) together with $H_{3}^{2}$ generate all the 1-qutrit classical gates. It is clear that we only need to construct $P[i]_{3}$ for some $i$. Without loss of generality, we assume the gate from 3 ) is $Q[2]_{3}$, since we can permute the basis elements with the classical 1-qutrit gates. From the identity $P[2]_{3}=Q[2]_{3}$ Flip $[2]_{3}$, it suffices to construct Flip $[2]_{3}$. The construction of the sign-flip gate was given as an exercise in [15] and a detailed proof can be found in Section 2.5 of [8]. For completeness, we also give the proof in Lemma 5 .

Lemma 5. [15], 8] The gate Flip[2] can be constructed probabilistically. Moreover, the probability to construct Flip[2] approaches to 1 exponentially fast in the number of gates and measurements given in Theorem 2 . 
Proof It's not hard to see that with the gates and measurement from Theorem 2, the following states and operations can be implemented.

1). $\widetilde{|i\rangle}=\frac{1}{\sqrt{3}} \sum_{j=0}^{2} \omega^{i j}|j\rangle=H|i\rangle, i=0,1,2$.

2). Projection of a 1-qutrit state to any computational basis vector, preserving the coherence of the orthogonal complement. For example, projection to $\operatorname{span}\{|2\rangle\}$ and its complement $\operatorname{span}\{|0\rangle,|1\rangle\}$.

$3)$. Measurement of a qutrit in the standard computational basis.

4). Projection to $\operatorname{span}\{\widetilde{|1\rangle}, \widetilde{|2\rangle}\}$ and its complement $\operatorname{span}\{\widetilde{|0\rangle}\}$

To obtain Flip[2], we first construct the ancilla $|\psi\rangle=\frac{1}{\sqrt{3}}(|0\rangle-|1\rangle+$ $|2\rangle)$ as follows.

Prepare the state $\widetilde{|1\rangle|\widetilde{2}\rangle}$, and project each qutrit to the space $\operatorname{span}\{|0\rangle,|1\rangle\}$ to obtain the state $|\eta\rangle=\frac{1}{2}(|0\rangle+\omega|1\rangle) \otimes\left(|0\rangle+\omega^{2}|1\rangle\right)$. Apply the $S U M$ gate to $|\eta\rangle$ and then project the first qutrit of the resulting state to the space $\operatorname{span}\{\widetilde{|0\rangle}\}$. It's easy to see on the second qutrit we get the state $|\psi\rangle$.

Now for a state $|\phi\rangle=c_{0}|0\rangle+c_{1}|1\rangle+c_{2}|2\rangle$, apply the $S U M$ gate to $|\phi\rangle|\psi\rangle$ and then measure the second qutrit in the standard basis. If the outcome is $|0\rangle$, then the first qutrit is $c_{0}|0\rangle+c_{1}|1\rangle-c_{2}|2\rangle$. If the outcome is $|1\rangle$, then the first qutrit is $-c_{0}|0\rangle+c_{1}|1\rangle+c_{2}|2\rangle$, and if the outcome is $|2\rangle$, then the first qutrit is $c_{0}|0\rangle-c_{1}|1\rangle+c_{2}|2\rangle$. Moreover, the probability for each case is $\frac{1}{3}$. Therefore, this process changes the sign of some coefficient randomly. Repeat this process until we get the gate Flip[2]. Note that we will also stop repeating the process if we obtain the gate Flip[0]* Flip[1], which is the same as Flip[2] up to a global sign.

Let $p_{n}$ be the probability that Flip[2] is obtained (up to a global sign) with no more than $n$ times of the process. It's not hard to derive a recursive formula for $p_{n}$ :

$$
p_{n}=p_{n-1}+\frac{1}{3}\left(1-p_{n-1}\right)=\frac{1}{3}+\frac{2}{3} p_{n-1}, \quad p_{1}=\frac{1}{3} .
$$

Therefore, we have $p_{n}=1-\left(\frac{2}{3}\right)^{n}$, which approximates to 1 exponentially fast.

\subsection{A universal qupit gate set.}

Theorem 3. The following gate set is universal for the qupit quantum circuit model for $p \geq 5$ :

1). The generalized Hadamard gate $H_{p}$

2). The SUM gate $\mathrm{SUM}_{p}$ 
3). The gates $Q[i]_{p}, i=1, \cdots, p-1$.

Remark 3. Note that the gate $Q[0]_{p}$ can be constructed from $Q[i]_{p}, i=$ $1, \cdots, p-1$, since $\prod_{i=0}^{p-1} Q[i]_{p}=\omega_{p} I d$.

Proof The proof is analogous to that of Theorem 1. By Lemma 8 below, the gates in 1) and 3) generate a a dense subgroup of $S U(p)$. By Lemma 3, $\mathrm{SUM}_{p}$ is an imprimitive gate. Again by Theorem 1.3 in [6], this is a universal gate set.

Corollary 1. $H_{p}, \mathrm{SUM}_{p}$ and all the 1-qupit classical gates, together with some $Q[i]_{p}$, form a universal qupit gate set.

The rest of this section is devoted to a proof of Lemma 8 .

Set $X[i]=H_{p} Q[i]_{p} H_{p}^{-1} Q[i]_{p}^{-1}, Y[i]=H_{p} Q[i]_{p}^{-1} H_{p}^{-1} Q[i]_{p}, i=0, \cdots, p-$ 1. And define

$$
S_{i}=\operatorname{span}\left\{|i\rangle, \sum_{j \neq i} \omega_{p}^{i j}|j\rangle\right\}
$$

Lemma 6. [3] $X[i], Y[i]$ act as the identity on $S_{i}^{\perp}$, and are of infinite order confined in $S_{i}$. Moreover, they do not commute.

Remark 4. It is worth noting that Lemma 6 does not hold for $d=3$. In the case of $d=3$, we need to replace $Q[i]$ by its square root $P[i]$. This is how we defined $W[i], Z[i]$ in Subsection 2.1,

Given a subspace $A \subset \mathbb{C}^{d}, S U(A)$ is identified with the subgroup of $S U\left(\mathbb{C}^{d}\right)$ whose elements are the identity on the orthogonal complement of $A$.

Lemma 7. 3. If $A, B$ are two non-orthogonal subspaces of $\mathbb{C}^{p}$, then $S U(A), S U(B)$ generate a dense subgroup of $S U(A+B)$.

Lemma 8. The generalized Hadamard gate $H_{p}$ and the gate set $\left\{Q[i]_{p}, i=\right.$ $0, \cdots, p-1\}$ generate a dense subgroup of $S U(p)$.

Proof By Lemma 6 and Lemma 1, $X[i]$ and $Y[i]$ generate a dense subgroup of $S U\left(S_{i}\right)$. It's easy to see that $S_{i}$ is not orthogonal to $\sum_{j=0}^{i-1} S_{j}$, and $\sum_{j=0}^{p-1} S_{j}=\mathbb{C}^{p}$. By induction on $i$ and by Lemma 7, we obtain a dense subgroup of $S U(p)$. 


\section{Universal Models from Metaplectic Anyon Systems}

We will follow the set-up of anyonic quantum computing models as in [25, 8, in particular the notations in Section 2 of [8]. We refer to a particular anyonic model by a pair $(V, b)$, where $V$ is the fusion space that encodes one qudit and $b$ a basis of $\mathrm{V}$ designated as the computational basis. This notation is not complete because we also need to specify the encoding of two qudits, which will be clear from the context.

3.1. The metaplectic anyon system $S O(p)_{2}$. For a detailed discussion of $S O(p)_{2}$, see [13]. The UMC $S O(p)_{2}$ for an odd prime $p=$ $2 r+1 \geq 5$ has $r+4$ isomorphism classes of simple objects (also called anyon types). We denote the set of simple object representatives by $\left\{\mathbf{1}, Z, X_{\epsilon}, X_{\epsilon}^{\prime}, Y_{j}, 1 \leq j \leq r\right\}$, and their types $\left\{1, z, \epsilon, \epsilon^{\prime}, y_{j}, 1 \leq j \leq r\right\}$. Their quantum dimensions are $d_{1}=d_{Z}=1, d_{X_{\epsilon}}=d_{X_{\epsilon^{\prime}}}=\sqrt{p}, d_{Y_{j}}=2$. We will follow [12] to refer to the anyons $X_{\epsilon}, X_{\epsilon}^{\prime}$ as the metaplectic anyons, and the anyons $\left\{Y_{j}, 1 \leq j \leq r\right\}$ as the metaplectic modes.

The following is a list of some of the fusion rules which are suffcient for deducing all the other fusion rules.

1). $X_{\epsilon} \otimes X_{\epsilon} \cong \mathbf{1} \oplus \oplus_{j=1}^{r} Y_{j}$

2). $X_{\epsilon} \otimes X_{\epsilon}^{\prime} \cong Z \oplus \oplus_{j=1}^{r} Y_{j}$

3). $X_{\epsilon} \otimes Y_{j} \cong X_{\epsilon} \oplus X_{\epsilon}^{\prime}, 1 \leq j \leq r$

4). $X_{\epsilon} \otimes Z \cong X_{\epsilon}^{\prime}$

5). $Z \otimes Z \cong 1$

6). $Z \otimes Y_{j} \cong Y_{j}, 1 \leq j \leq r$

7). $Y_{j} \otimes Y_{j} \cong \mathbf{1} \oplus Z \oplus Y_{\min \{2 j, m-2 j\}}, 1 \leq j \leq r$

8). $Y_{i} \otimes Y_{j} \cong Y_{|i-j|} \oplus Y_{\min \{i+j, m-i-j\}}, 1 \leq i, j \leq r, i \neq j$

The UMC $S O(3)_{2}$ is the same as $S U(2)_{4}$. There are five anyon types in $S O(3)_{2}$, namely, $1, z, \epsilon, \epsilon^{\prime}, y$. Their quantum dimensions are $1,1, \sqrt{3}, \sqrt{3}, 2$.

Remark 5. The anyon types in $S U(2)_{4}$ are usually denoted as $\{0,1,2,3,4\}$, which are twice the spin of the corresponding irreps of $S U(2)$. The correspondence between $S O(3)_{2}$ and $S U(2)_{4}$ labels are given as follows:

$1 \leftrightarrow 0, \quad z \leftrightarrow 4, \quad \epsilon \leftrightarrow 1, \quad \epsilon^{\prime} \leftrightarrow 3, \quad y \leftrightarrow 2$,

We use the fusion tree shown in Figure 1 to encode a qutrit.

The associated Hilbert space $V_{y}^{\epsilon \epsilon \epsilon \epsilon}$ is 3-dimensional with the computational basis $\{-|Y Y\rangle,|\mathbf{1} Y\rangle,|Y \mathbf{1}\rangle\}$. We will often use the type labels in fusion spaces. The computational basis $\{-|Y Y\rangle,|\mathbf{1} Y\rangle,|Y \mathbf{1}\rangle\}$ is identified with the qutrit basis $\{|0\rangle,|1\rangle,|2\rangle\}$, respectively. Note that this qutrit basis does not have a $\mathbb{Z}_{3}$ symmetry. We will denote this $S U(2)_{4}$ 


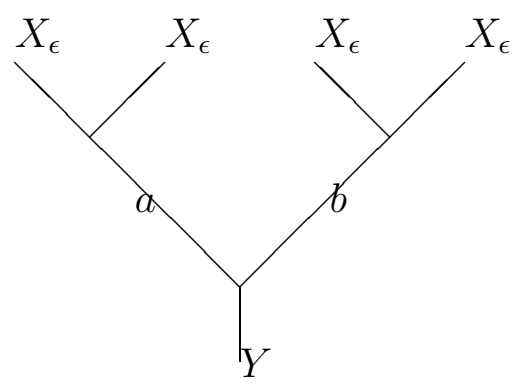

FIGURE 1. 1-qutrit model

anyonic computational model as $\left(V_{y}^{\epsilon \epsilon \epsilon \epsilon},\{-|Y Y\rangle,|\mathbf{1} Y\rangle,|Y \mathbf{1}\rangle\}\right)$ or simply $V_{y}^{\epsilon \epsilon \epsilon \epsilon}$.

Remark 6. The minus sign in front of $|Y Y\rangle$ is introduced to make the braid representation matrices into a nicer form. This is inessential since the gate Flip[0] that changes the sign of $|0\rangle$ can be constructed.

The data needed to analyze the computational power of our model are the $F$-matrices and $R$-symbols. All the $F$-matrices and $R$-symbols for $S U(2)_{4}$ are listed in Appendix A. Partial data enough for our purpose for $S O(5)_{2}$ are listed in Appendix B. See Appendix Cl on how braid matrices for $\sigma_{1}, \sigma_{2}, \sigma_{3}$ in the following subsections are derived.

3.2. The universal model $V_{y}^{\epsilon \epsilon \epsilon \epsilon}$ with $S U(2)_{4}$. Under the basis $\{-|Y Y\rangle,|\mathbf{1} Y\rangle,|Y \mathbf{1}\rangle\}$, the generators of the braid group $\mathcal{B}_{4}$ for the representation $V_{y}^{\epsilon \epsilon \epsilon \epsilon}$ have the following matrices:

$$
\begin{aligned}
& \sigma_{1}=\gamma\left(\begin{array}{ccc}
1 & 0 & 0 \\
0 & \omega & 0 \\
0 & 0 & 1
\end{array}\right) \\
& \sigma_{2}=\gamma\left(\begin{array}{cccc}
\frac{1}{2}+\frac{\sqrt{3} i}{6} & -\frac{1}{2}+\frac{\sqrt{3} i}{6} & -\frac{1}{2}+\frac{\sqrt{3} i}{6} \\
-\frac{1}{2}+\frac{\sqrt{3} i}{6} & \frac{1}{2}+\frac{\sqrt{3} i}{6} & -\frac{1}{2}+\frac{\sqrt{3} i}{6} \\
0 & 1 & 0 \\
0 & 0 & \omega
\end{array}\right),
\end{aligned}
$$

where $\gamma=e^{\frac{\pi i}{12}}$.

Note that $\sigma_{1}, \sigma_{3}$ are just $Q[1]_{3}, Q[2]_{3}$ defined in Section 2 , up to a phase.

The group generated by these matrices is a subgroup of $S U(3)$ of order 648 whose center is isomorphic to $\mathbb{Z}_{3}$. It is isomorphic to the complex reflection group which is the 25 -th item in the classification 


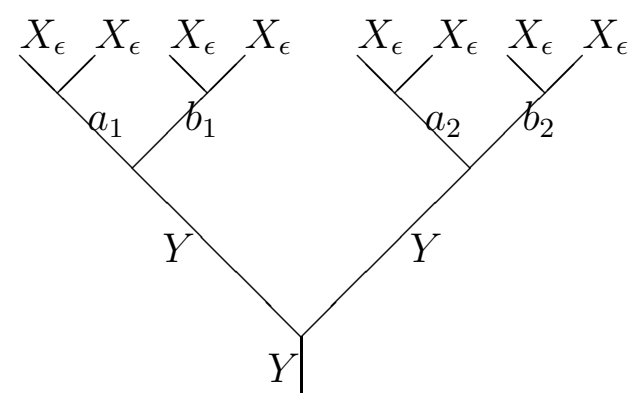

FiguRE 2. 2-qutrit model

table of finite complex reflection groups in [24]. The elements in the center are scalar matrices. And the group modulo the center is isomorphic to the famous Hessian group $\sum(216)$ of order 216, which is also the 1-qutrit Clifford group [14] [18] [9]. In the following, we will choose many braids whose representation matrices provide us desired gates. They are obtained by systematically analyzing the representation $V_{y}^{\epsilon \epsilon \epsilon \epsilon}$ of $\mathcal{B}_{4}$.

Define $p=\sigma_{1} \sigma_{2} \sigma_{1}, q=\sigma_{3} \sigma_{2} \sigma_{3}, H=q^{2} p q^{2}$, then (ignoring the phase $\gamma)$

$$
\begin{aligned}
& p^{2}=-\left(\begin{array}{lll}
0 & 1 & 0 \\
1 & 0 & 0 \\
0 & 0 & 1
\end{array}\right) \quad q^{2}=-\left(\begin{array}{lll}
0 & 0 & 1 \\
0 & 1 & 0 \\
1 & 0 & 0
\end{array}\right) \\
& H=\frac{1}{\sqrt{3} i}\left(\begin{array}{ccc}
1 & 1 & 1 \\
1 & \omega & \omega^{2} \\
1 & \omega^{2} & \omega
\end{array}\right)
\end{aligned}
$$

Thus, by braiding alone we obtained all the 1-qutrit classical gates, the generalized Hadamard gate and the gates $Q[i]_{3}, i=0,1,2$.

Next, we consider the encoding of the 2-qutrits using the 9 dimen-

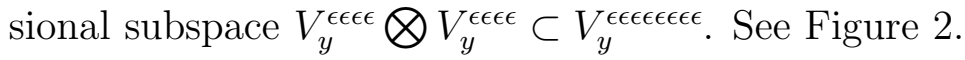

Let $s_{1}=\sigma_{2} \sigma_{1} \sigma_{3} \sigma_{2}, s_{2}=\sigma_{4} \sigma_{3} \sigma_{5} \sigma_{4}, s_{3}=\sigma_{6} \sigma_{5} \sigma_{7} \sigma_{6}$. And let $\bigwedge(Z)=$ $s_{1}^{-1} s_{2}^{2} s_{1} s_{3}^{-1} s_{2}^{2} s_{3}$.

It can be verified that $\bigwedge(Z)$, restricted to the 9-dimensional subspace $V_{y}^{\epsilon \epsilon \epsilon \epsilon} \bigotimes V_{y}^{\epsilon \epsilon \epsilon \epsilon} \subset V_{y}^{\epsilon \epsilon \epsilon \epsilon \epsilon \epsilon \epsilon \epsilon}$, is exactly the Controlled- $Z$ gate when $\{-|Y Y\rangle,|\mathbf{1} Y\rangle,|Y \mathbf{1}\rangle\}$ is the computational basis for each qutrit $V_{y}^{\epsilon \epsilon \epsilon \epsilon}$. By drawing the braids $s_{i}, i=1,2,3$, it is not hard to be convinced that this Controlled- $Z$ gate has no leakage. Therefore, our anyonic model is leakage-free. 
Recall the definitions from the beginning of Section 2, we see that the SUM gate is related to the Controlled- $Z$ gate through the generalized Hadamard gate $H$. Explicitly, we have

$$
\mathrm{SUM}=(I d \otimes H) \bigwedge(Z)^{-1}\left(I d \otimes H^{-1}\right) .
$$

Thus, we can obtain the SUM gate by braiding, since $H$ is already a braiding circuit. We have:

Proposition 1. By braiding alone, we can construct the classical 1qutrit gates, the generalized Hadamard gate, the generalized $\sigma_{z}$ gates $Q[i]$, and the SUM gate for our anyonic model $V_{y}^{\epsilon \epsilon \epsilon \epsilon}$.

It follows from Theorem 1 that we need to find the square roots of $Q[i]$ to make our model universal. Our solution is to introduce a physically realistic measurement: to determine wether or not the total charge of two anyons is trivial.

Measurement 2. Let $\mathcal{M}_{1}=\left\{\Pi_{1}, \Pi_{1}^{\prime}\right\}$ be the projective measurement onto the total charge $=\mathbf{1}$ sector of two anyons and its complement. Then $\mathcal{M}_{1}$ allows us to distinguish between the trivial anyon $\mathbf{1}$ and other anyons; namely, check whether an anyon is trivial or not. Moreover, in a 1-qutrit model, the state of the second pair after each outcome of the measurement of the first pair is still coherent.

Applying Measurement 2 to the first two anyons in the 1-qutrit model to determine their total charge is equivalent to projecting the state to the subspace $\operatorname{span}\{|\mathbf{1} Y\rangle\}$ and its orthogonal complement $\operatorname{span}\{-|Y Y\rangle,|Y \mathbf{1}\rangle\}$. Since all the 1-qutrit classical gates can be constructed by braiding, we can also project the state to $\operatorname{span}\{-|Y Y\rangle\}$ and $\operatorname{span}\{|\mathbf{1} Y\rangle,|Y \mathbf{1}\rangle\}$. Thus, Measurement 1 can be obtained from Measurement 2 and braiding. It is important to notice that when the total charge of the first two anyons of a qutrit is $Y$, then the total charge of the second pair of anyons is in a coherent superposition of $\mathbf{1}$ and $Y$.

Another method to measure total charge of anyons is interferometric measurement. It is known that any projective measurement of total charge of anyons can be simulated by interferometric measurements [11.

Therefore, by braiding anyons and Measurement 2, we can construct the generalized Hadamard gate $H$, the SUM gate, all the $Q[i]$ 's, all the 1-qutrit classical gates and Measuremnt 11. These are exactly the universal gate set in Theorem 2 .

Theorem 4. In the $S U(2)_{4}$ theory, if we use the fusion space of four metaplectic anyons $X_{\epsilon}$ with total charge $Y$ as a 1-qutrit (See 1), and 


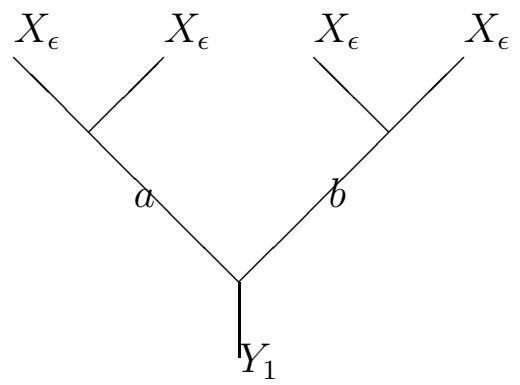

FiguRE 3. 1-qupit model

choose $\{-|Y Y\rangle,|\mathbf{1} Y\rangle,|Y \mathbf{1}\rangle\}$ as the computational basis, then braiding supplemented with Measurement 2 of two metaplectic anyons forms a universal gate set for our anyonic quantum computation.

Proof It follows from Prop. 1 and Theorem 2.

The most challenging part of our model is to maintain the total charge of many metaplectic anyons to be the meteplectic mode $Y$. We cannot make the more natural choice of total charge trivial model universal, and provide evidence below that this cannot be done.

3.3. A model from $S O(5)_{2}$. The $S O(5)_{2}$ theory consists of six anyon types: $\left\{1, z, y_{1}, y_{2}, \epsilon, \epsilon^{\prime}\right\}$. We set up a similar model as that for $S O(3)_{2}$.

For a 1-qupit $p=5$, use the model as shown in Figure 3 .

The Hilbert space $V_{y_{1}}^{\epsilon \epsilon \epsilon}$ now is 5-dimensional with the computational basis $\left\{\left|Y_{2} Y_{2}\right\rangle,\left|\mathbf{1} Y_{1}\right\rangle,\left|Y_{2} Y_{1}\right\rangle,\left|Y_{1} Y_{2}\right\rangle,\left|Y_{1} \mathbf{1}\right\rangle\right\}$. The representation matrices of the generators of $\mathcal{B}_{4}$ are:

$$
\begin{aligned}
\sigma_{1} & =\frac{1}{i}\left(\begin{array}{ccccc}
e^{\frac{2 i \pi}{5}} & 0 & 0 & 0 & 0 \\
0 & 1 & 0 & 0 & 0 \\
0 & 0 & e^{\frac{2 i \pi}{5}} & 0 & 0 \\
0 & 0 & 0 & e^{-\frac{2 i \pi}{5}} & 0 \\
0 & 0 & 0 & 0 & e^{-\frac{2 i \pi}{5}}
\end{array}\right) \\
\sigma_{2} & =\frac{1}{\sqrt{5} i}\left(\begin{array}{ccccc}
1 & e^{-\frac{2 i \pi}{5}} & e^{\frac{2 i \pi}{5}} & e^{\frac{2 i \pi}{5}} & e^{-\frac{2 i \pi}{5}} \\
e^{-\frac{2 i \pi}{5}} & 1 & e^{-\frac{2 i \pi}{5}} & e^{\frac{2 i \pi}{5}} & e^{\frac{2 i \pi}{5}} \\
e^{\frac{2 i \pi}{5}} & e^{-\frac{2 i \pi}{5}} & 1 & e^{-\frac{2 i \pi}{5}} & e^{\frac{2 i \pi}{5}} \\
e^{\frac{2 i \pi}{5}} & e^{\frac{2 i \pi}{5}} & e^{-\frac{2 i \pi}{5}} & 1 & e^{-\frac{2 i \pi}{5}} \\
e^{-\frac{2 i \pi}{5}} & e^{\frac{2 i \pi}{5}} & e^{\frac{2 i \pi}{5}} & e^{-\frac{2 i \pi}{5}} & 1
\end{array}\right)
\end{aligned}
$$




$$
\sigma_{3}=\frac{1}{i}\left(\begin{array}{ccccc}
e^{\frac{2 i \pi}{5}} & 0 & 0 & 0 & 0 \\
0 & e^{-\frac{2 i \pi}{5}} & 0 & 0 & 0 \\
0 & 0 & e^{-\frac{2 i \pi}{5}} & 0 & 0 \\
0 & 0 & 0 & e^{\frac{2 i \pi}{5}} & 0 \\
0 & 0 & 0 & 0 & 1
\end{array}\right)
$$

The representation $V_{y_{1}}^{\epsilon \epsilon \epsilon}$ of the braid group $\mathcal{B}_{4}$ is irreducible and the image is the Clifford group, which is isomorphic to $\left(\mathbb{Z}_{5} \times \mathbb{Z}_{5}\right) \rtimes \mathrm{SL}\left(2, \mathbb{Z}_{5}\right)$.

Direct calculations lead to the following important gates, up to a phase, from braiding.

The generalized Hadamard gate $H_{5}: H_{5}|j\rangle=\sum_{i=0}^{4} \omega_{5}^{i j}|i\rangle,=\sigma_{1}^{-1} \sigma_{3}^{-1} \sigma_{2}^{2} \sigma_{1}^{-1} \sigma_{3}^{-1}$.

The generalized $Z$-gate $Z: Z|i\rangle=\omega_{5}^{i}|i\rangle=\sigma_{1} \sigma_{3}^{-1}$.

The generalized $X$-gate $X: X|i\rangle=|i+1\rangle=\sigma_{1} \sigma_{2} \sigma_{1}^{-2} \sigma_{3}^{2}\left(\sigma_{1} \sigma_{2}\right)^{-1}$.

The multiplication gate $M[k]: M[k]|i\rangle=|k i\rangle, k=2,3,4$. These gates are realized by $\sigma_{1}^{2} \sigma_{2}^{-2} \sigma_{1}^{-1} \sigma_{3}^{-1} \sigma_{2} \sigma_{1}, \sigma_{1}^{2} \sigma_{2}^{-1} \sigma_{1} \sigma_{3} \sigma_{2}^{2} \sigma_{3}$, and $\sigma_{1} \sigma_{2} \sigma_{1} \sigma_{3} \sigma_{2} \sigma_{1}$.

The gates $X$ and $M[k]$ are classical 1-qupit gates, i.e., permutation matrices. Identifying a permutation matrix with the permutation that it represents in the permutation group $\mathcal{S}_{5}$, we see that $X, M[k]$ generate a maximal subgroup of $\mathcal{S}_{5}$ with order 20 , which is isomorphic to $\mathbb{Z}_{5} \rtimes \mathbb{Z}_{4}$. Moreover, this maximal subgroup contains all the 1-qupit classical gates obtained from braiding. Since this subgroup is maximal, any classical gate out of the subgroup is enough to produce all the 1-qupit classical gates. We speculate that Measurement 2 would help produce an extra classical gate.

The 2-qupit encoding is also analogous to the one we used in $S O(3)_{2}$. See Figure 4 .

We obtain the Controlled- $Z$ gate $\Lambda(Z)$ by the same braiding as we did in Subsection 3.2. Here $\bigwedge(Z)|i, j\rangle=\omega_{5}^{i j}|i, j\rangle$. And again, the SUM gate is obtained by conjugating $\bigwedge(Z)$ by the generalized Hadamard $H$.

Proposition 2. The gates that can be constructed from braiding include the generalized Hadamard $H, 20$ 1-qupit classical gates generated by the generalized $X$ gate and multiplication gates $M[k]$ 's, the generalized $Z$ gate and the SUM gate. In view of Corollary 1, we need an extra 1-qupit classical gate and some gate $Q[i]$ to make this model universal. 


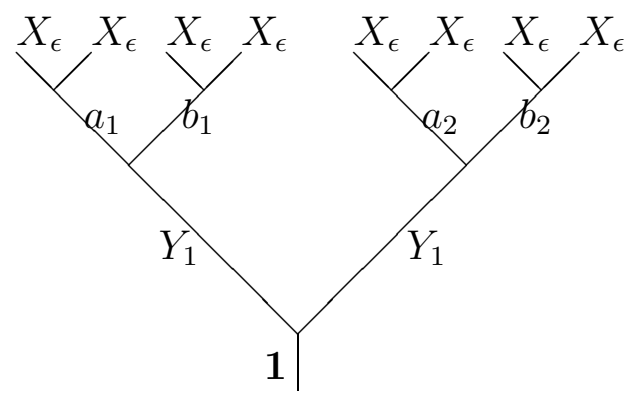

Figure 4. 2-qupit model

Suppose we have all the 1-qupit classical gates, then clearly by using $\sigma_{1}$ and classical gates, we can obtain the gates $R[i, j, k]=\left(Q[i] Q[j]^{-1}\right)^{k}, i \neq$ $j, k=1,2,3,4$. For example,

$$
R[1,2, k]=\left(\begin{array}{ccccc}
\omega^{k} & 0 & 0 & 0 & 0 \\
0 & \omega^{-k} & 0 & 0 & 0 \\
0 & 0 & 1 & 0 & 0 \\
0 & 0 & 0 & 1 & 0 \\
0 & 0 & 0 & 0 & 1
\end{array}\right)
$$

Let $X[i, j, k]=H R[i, j, k] H^{-1} R[i, j, k]^{-1}$,

It can be shown that $X[i, j, k]$ is of infinite order, and for fixed $i, j$, the four matrices $\{X[i, j, k], k=1,2,3,4\}$ fix some 1-dimensional subspace and act irreducibly on the 4-dimensional orthogonal complement. For example, $\{X[1,2, k], k=1,2,3,4\}$ fix the vector $\omega^{-1}|2\rangle+\frac{\sqrt{5}+1}{2} \omega^{2}|3\rangle+$ $|4\rangle$. If one can show $\{X[i, j, k], k=1,2,3,4\}$ generate a dense subgroup of the unitary group of the 4-dimensional complement for some $i \neq$ $j$, then it is straightforward to prove the gate set generate a dense subgroup of $S U(5)$ by Lemma 2. We did not succeed in showing this either.

3.4. Other models with $S U(2)_{4}$. There are at least 4 obvious anyonic quantum computing models with $S U(2)_{4}$ anyons. Besides the universal model that we studied, three others are the qubit model $V_{0}^{1111}$, the qutrit model $V_{0}^{2222}$, and the qubit model $V_{0}^{1221}$. The computational power of the corresponding models $V_{0}^{2222}$ and $V_{0}^{1221}$ in the JonesKauffman version of $S U(2)_{4}$ is analyzed in [17]. We conjecture that the model $V_{0}^{1111}$, shown in Figure 5, with measurements of total charges of 


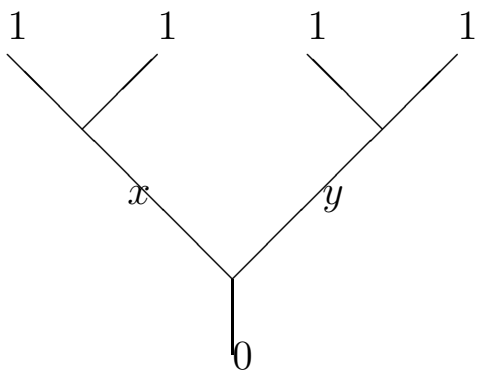

FiguRE 5. 1-qubit model

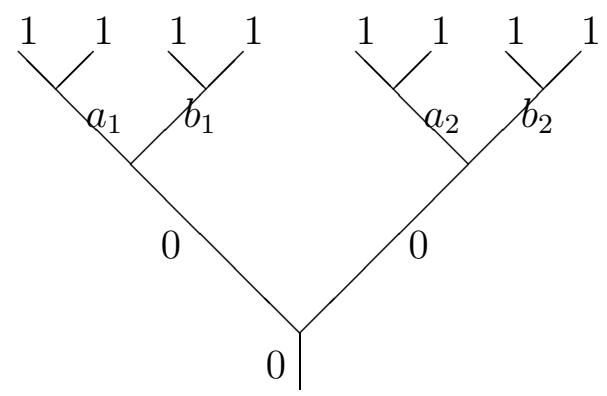

FiguRE 6. 2-qubit model

metaplectic anyons is Ising like, i.e., braidings and such measurements can be simulated classically efficiently.

The Hilbert space $V_{0}^{1111}$ is 2-dimensional with basis $\{|00\rangle,|22\rangle\}$. Under this basis, the $\sigma_{i}{ }^{\prime} s$ have the following matrices:

$$
\sigma_{1}=\sigma_{3}=\gamma\left(\begin{array}{ll}
\omega & 0 \\
0 & 1
\end{array}\right) \quad \sigma_{2}=\gamma \bar{\omega}\left(\begin{array}{cc}
-\frac{1}{2}+\frac{\sqrt{3} i}{6} & \frac{\sqrt{6} i}{3} \\
\frac{\sqrt{6} i}{3} & -\frac{1}{2}-\frac{\sqrt{3} i}{6}
\end{array}\right) \text {, for some }
$$

phase $\gamma$.

Up to normalization, this representation is the same as one component of $V_{B}^{D D D D}$ in $D\left(S_{3}\right)$, where the two components of $V_{B}^{D D D D}$ are isomorphic. See Appendix B.2.2 in [8].

These matrices generate a group of size 24 which is isomorphic to $\operatorname{SL}\left(2, \mathbb{F}_{3}\right)$. Modulo the center, we get the even permutations $\mathcal{A}_{4}$.

Similarly, for the 2-qubit encoding as that in Figure 6 .

We use $\left|0 ; a_{1} b_{1}\right\rangle \otimes\left|0 ; a_{2} b_{2}\right\rangle$ to denote the state in Figure 6 . 
Then the same braiding as before gives the following transformation: $|0 ; 00\rangle \otimes|0 ; 00\rangle \mapsto|0 ; 00\rangle \otimes|0 ; 00\rangle$, $|0 ; 00\rangle \otimes|0 ; 22\rangle \mapsto|0 ; 00\rangle \otimes|0 ; 22\rangle$, $|0 ; 22\rangle \otimes|0 ; 00\rangle \mapsto|0 ; 22\rangle \otimes|0 ; 00\rangle$, $|0 ; 22\rangle \otimes|0 ; 22\rangle \mapsto-\frac{1}{2}|0 ; 22\rangle \otimes|0 ; 22\rangle+\frac{\sqrt{3} i}{2}|4 ; 22\rangle \otimes|4 ; 22\rangle$

Thus, projecting out the charge 4 , we obtain the Controlled- $\sigma_{z}$ gate $\bigwedge\left(\sigma_{z}\right)$. But when the state is actually projected onto the charge 4 part, the state is destroyed and the whole computational process has to start over again.

\section{Appendix A. F-Matrices AND $R$-Symbols fOR $S U(2)_{4}$}

We order the labels $\{0,1,2,3,4\}$ in increasing order when we arrange the entries of the $F$-matrices. The conventions that we used for $F$ matrices and $R$-symbols are in [8]. We drop all trivial $F_{d}^{a b c}=1$ when $a, b, c, d$ is admissible, in particular when one of $a, b$ or $c$ is trivial.

- $(-1)$ for

$F_{4}^{114}, F_{4}^{123}, F_{3}^{124}, F_{4}^{132}, F_{3}^{133}, F_{2}^{134}, F_{4}^{141}, F_{3}^{142}, F_{2}^{143}, F_{1}^{144}, F_{4}^{213}, F_{3}^{214}$

$F_{4}^{222}, F_{2}^{224}, F_{4}^{231}, F_{1}^{234}, F_{3}^{241}, F_{2}^{242}, F_{1}^{243}, F_{4}^{312}, F_{3}^{313}, F_{2}^{314}, F_{4}^{321}, F_{1}^{324}$

$F_{3}^{331}, F_{1}^{333}, F_{4}^{334}, F_{2}^{341}, F_{1}^{342}, F_{4}^{343}, F_{3}^{344}, F_{4}^{411}, F_{3}^{412}, F_{2}^{413}, F_{1}^{414}, F_{3}^{421}$

$F_{2}^{422}, F_{1}^{423}, F_{2}^{431}, F_{1}^{432}, F_{4}^{433}, F_{3}^{434}, F_{1}^{441}, F_{3}^{443}$

- $\left(\begin{array}{cc}-\frac{1}{\sqrt{3}} & \sqrt{\frac{2}{3}} \\ \sqrt{\frac{2}{3}} & \frac{1}{\sqrt{3}}\end{array}\right)$ for $F_{1}^{111}, F_{3}^{131}, F_{1}^{313}, F_{3}^{333}$

- $\left(\begin{array}{cc}-\frac{1}{\sqrt{2}} & \frac{1}{\sqrt{2}} \\ \frac{1}{\sqrt{2}} & \frac{1}{\sqrt{2}}\end{array}\right)$ for

$F_{2}^{112}, F_{1}^{122}, F_{3}^{122}, F_{2}^{132}, F_{2}^{211}, F_{2}^{213}, F_{1}^{221}, F_{3}^{221}, F_{1}^{223}, F_{2}^{231}, F_{2}^{312}, F_{1}^{322}$

- $\left(\begin{array}{cc}-\sqrt{\frac{2}{3}} & \frac{1}{\sqrt{3}} \\ \frac{1}{\sqrt{3}} & \sqrt{\frac{2}{3}}\end{array}\right)$ for $F_{3}^{113}, F_{1}^{133}, F_{3}^{311}, F_{1}^{331}$

- $\left(\begin{array}{cc}-\frac{1}{2} & \frac{\sqrt{3}}{2} \\ \frac{\sqrt{3}}{2} & \frac{1}{2}\end{array}\right)$ for $F_{2}^{121}, F_{1}^{212}$

- $\left(\begin{array}{cc}-\frac{\sqrt{3}}{2} & \frac{1}{2} \\ \frac{1}{2} & \frac{\sqrt{3}}{2}\end{array}\right)$ for $F_{2}^{123}, F_{3}^{212}, F_{1}^{232}, F_{2}^{321}$

- $\left(\begin{array}{cc}\frac{1}{\sqrt{2}} & -\frac{1}{\sqrt{2}} \\ -\frac{1}{\sqrt{2}} & -\frac{1}{\sqrt{2}}\end{array}\right)$ for $F_{3}^{223}, F_{2}^{233}, F_{3}^{322}, F_{2}^{332}$

- $\left(\begin{array}{cc}\frac{1}{2} & -\frac{\sqrt{3}}{2} \\ -\frac{\sqrt{3}}{2} & -\frac{1}{2}\end{array}\right)$ for $F_{3}^{232}, F_{2}^{323}$ 


$$
\text { - }\left(\begin{array}{ccc}
\frac{1}{2} & -\frac{1}{\sqrt{2}} & \frac{1}{2} \\
-\frac{1}{\sqrt{2}} & 0 & \frac{1}{\sqrt{2}} \\
\frac{1}{2} & \frac{1}{\sqrt{2}} & \frac{1}{2}
\end{array}\right) \text { for } F_{2}^{222}
$$

\section{A.1. $R$-symbols.}

- 1 for $R_{0}^{00}, R_{1}^{01}, R_{2}^{02}, R_{3}^{03}, R_{4}^{04}, R_{1}^{10}, R_{2}^{20}, R_{3}^{30}, R_{4}^{40}, R_{0}^{44}$

- $e^{\frac{3 i \pi}{4}}$ for $R_{0}^{11}$

- $e^{\frac{i \pi}{12}}$ for $R_{2}^{11}$

- $e^{\frac{2 i \pi}{3}}$ for $R_{1}^{12}, R_{1}^{21}, R_{2}^{22}, R_{3}^{23}, R_{3}^{32}$

- $e^{\frac{i \pi}{6}}$ for $R_{3}^{12}, R_{3}^{21}$

- $e^{\frac{7 i \pi}{12}}$ for $R_{2}^{13}, R_{2}^{31}$

- $e^{\frac{i \pi}{4}}$ for $R_{4}^{13}, R_{4}^{31}$

- $i$ for $R_{3}^{14}, R_{3}^{41}$

- $e^{-\frac{2 i \pi}{3}}$ for $R_{0}^{22}$

- $e^{\frac{i \pi}{3}}$ for $R_{4}^{22}$

- $e^{-\frac{5 i \pi}{6}}$ for $R_{1}^{23}, R_{1}^{32}$

- -1 for $R_{2}^{24}, R_{2}^{42}$

- $e^{-\frac{i \pi}{4}}$ for $R_{0}^{33}$

- $e^{-\frac{11 i \pi}{12}}$ for $R_{2}^{33}$

- $-i$ for $R_{1}^{34}, R_{1}^{43}$

Appendix B. F-Matrices And $R$-SymbOls fOR $S O(5)_{2}$

Here we list all the $6 j$ symbols and some of the $R$-symbols that we need in this paper for the theory $S O(5)_{2}$. Again we omit the trivial $F_{d}^{a b c}$. We arrange the label set in the order $\left\{1, z, y_{1}, y_{2}, \epsilon, \epsilon^{\prime}\right\}$ in the following.

$$
\begin{aligned}
& \text { Let } h=\sqrt{10-2 \sqrt{5}}, k=\sqrt{10+2 \sqrt{5}} \text {. } \\
& \text { - }(-1) \text { for } \\
& F_{y_{2}}^{z y_{1} y_{1}}, F_{y_{1}}^{z y_{1} y_{2}}, F_{y_{1}}^{z y_{2} y_{1}}, F_{y_{2}}^{z y_{2} y_{1}}, F_{\epsilon}^{z \epsilon z}, F_{\epsilon^{\prime}}^{z \epsilon y_{1}}, F_{\epsilon^{\prime}}^{z \epsilon y_{2}}, F_{\epsilon^{\prime}}^{z \epsilon^{\prime} z}, F_{\epsilon}^{z \epsilon^{\prime} y_{1}} \\
& F_{\epsilon}^{z \epsilon^{\prime} y_{2}}, F_{y_{2}}^{y_{1} z y_{1}}, F_{y_{1}}^{y_{1} z y_{2}}, F_{y_{2}}^{y_{1} y_{1} z}, F_{z}^{y_{1} y_{1} y_{2}}, F_{y_{1}}^{y_{1} y_{2} z}, F_{y_{2}}^{y_{1} y_{2} z}, F_{z}^{y_{1} y_{2} y_{1}}, F_{\epsilon^{\prime}}^{y_{1} \epsilon z} \\
& F_{\epsilon}^{y_{1} \epsilon^{\prime} z}, F_{y_{1}}^{y_{2} z y_{1}}, F_{y_{1}}^{y_{2} z y_{2}}, F_{y_{1}}^{y_{2} y_{1} z}, F_{z}^{y_{2} y_{1} y_{1}}, F_{z}^{y_{2} y_{1} y_{2}}, F_{\epsilon^{\prime}}^{y_{2} \epsilon z}, F_{\epsilon}^{y_{2} \epsilon^{\prime} z}, F_{z}^{\epsilon z \epsilon} \\
& F_{y_{1}}^{\epsilon z \epsilon^{\prime}}, F_{y_{2}}^{\epsilon z \epsilon^{\prime}}, F_{z}^{\epsilon y_{1} \epsilon^{\prime}}, F_{z}^{\epsilon y_{2} \epsilon^{\prime}}, F_{y_{1}}^{\epsilon^{\prime} z \epsilon}, F_{y_{2}}^{\epsilon^{\prime} z \epsilon}, F_{z}^{\epsilon^{\prime} z \epsilon^{\prime}}, F_{z}^{\epsilon^{\prime} y_{1} \epsilon}, F_{z}^{\epsilon^{\prime} y_{2} \epsilon} \\
& \text { - }\left(\begin{array}{cc}
\frac{1}{\sqrt{2}} & -\frac{1}{\sqrt{2}} \\
\frac{1}{\sqrt{2}} & \frac{1}{\sqrt{2}}
\end{array}\right) \text { for } \\
& F_{y_{2}}^{y_{1} y_{1} y_{2}}, F_{\epsilon^{\prime}}^{y_{1} y_{1} \epsilon}, F_{\epsilon}^{y_{1} y_{1} \epsilon^{\prime}}, F_{y_{2}}^{y_{1} \epsilon \epsilon}, F_{y_{1}}^{y_{2} y_{2} y_{1}}, F_{y_{1}}^{y_{2} \epsilon \epsilon}, F_{\epsilon}^{\epsilon y_{1} y_{2}}, F_{\epsilon}^{\epsilon y_{2} y_{1}}, F_{y_{1}}^{\epsilon \epsilon_{1}^{\prime} y_{1}} \\
& F_{y_{1}}^{\epsilon^{\prime} \epsilon y_{1}} \\
& \text { - }\left(\begin{array}{cc}
\frac{1}{\sqrt{2}} & \frac{1}{\sqrt{2}} \\
\frac{1}{\sqrt{2}} & -\frac{1}{\sqrt{2}}
\end{array}\right) \text { for }
\end{aligned}
$$


UNIVERSAL QUANTUM COMPUTATION WITH METAPLECTIC ANYONS 19

$F_{\epsilon}^{y_{1} y_{1} \epsilon}, F_{\epsilon^{\prime}}^{y_{1} y_{1} \epsilon^{\prime}}, F_{y_{1}}^{y_{1} \epsilon \epsilon}, F_{y_{1}}^{y_{1} \epsilon^{\prime} \epsilon^{\prime}}, F_{\epsilon}^{y_{2} y_{2} \epsilon}, F_{\epsilon^{\prime}}^{y_{2} y_{2} \epsilon}, F_{\epsilon}^{y_{2} y_{2} \epsilon^{\prime}}, F_{\epsilon^{\prime}}^{y_{2} y_{2} \epsilon^{\prime}}, F_{y_{2}}^{y_{2} \epsilon \epsilon}$

$F_{y_{2}}^{y_{2} \epsilon \epsilon^{\prime}}, F_{y_{2}}^{y_{2} \epsilon^{\prime} \epsilon}, F_{y_{2}}^{y_{2} \epsilon^{\prime} \epsilon^{\prime}}, F_{\epsilon}^{\epsilon y_{1} y_{1}}, F_{\epsilon}^{\epsilon y_{2} y_{2}}, F_{\epsilon^{\prime}}^{\epsilon y_{2} y_{2}}, F_{y_{1}}^{\epsilon \epsilon y_{1}}, F_{y_{2}}^{\epsilon \epsilon y_{2}}, F_{y_{2}}^{\epsilon \epsilon^{\prime} y_{2}}$

$F_{\epsilon^{\prime}}^{\epsilon^{\prime} y_{1} y_{1}}, F_{\epsilon}^{\epsilon^{\prime} y_{2} y_{2}}, F_{\epsilon^{\prime}}^{\epsilon^{\prime} y_{2} y_{2}}, F_{y_{2}}^{\epsilon^{\prime} \epsilon y_{2}}, F_{y_{1}}^{\epsilon^{\prime} \epsilon^{\prime} y_{1}}, F_{y_{2}}^{\epsilon^{\prime} \epsilon^{\prime} y_{2}}$

- $\left(\begin{array}{ll}0 & 1 \\ 1 & 0\end{array}\right)$ for $F_{y_{2}}^{y_{1} y_{2} y_{1}}, F_{y_{1}}^{y_{2} y_{1} y_{2}}$

- $\left(\begin{array}{cc}\frac{1}{\sqrt{2}} & \frac{1}{\sqrt{2}} \\ -\frac{1}{\sqrt{2}} & \frac{1}{\sqrt{2}}\end{array}\right)$ for

$F_{y_{1}}^{y_{1} y_{2} y_{2}}, F_{\epsilon}^{y_{1} y_{2} \epsilon}, F_{y_{1}}^{y_{1} \epsilon \epsilon^{\prime}}, F_{y_{1}}^{y_{1} \epsilon^{\prime} \epsilon}, F_{y_{2}}^{y_{2} y_{1} y_{1}}, F_{\epsilon}^{y_{2} y_{1} \epsilon}, F_{\epsilon^{\prime}}^{\epsilon y_{1} y_{1}}, F_{y_{2}}^{\epsilon \epsilon y_{1}}, F_{y_{1}}^{\epsilon \epsilon y_{2}}$

$F_{\epsilon}^{\epsilon^{\prime} y_{1} y_{1}}$

- $\left(\begin{array}{cc}-\frac{1}{\sqrt{2}} & \frac{1}{\sqrt{2}} \\ \frac{1}{\sqrt{2}} & \frac{1}{\sqrt{2}}\end{array}\right)$ for

$F_{\epsilon^{\prime}}^{y_{1} y_{2} \epsilon}, F_{y_{2}}^{y_{1} \epsilon^{\prime} \epsilon}, F_{\epsilon}^{y_{2} y_{1} \epsilon^{\prime}}, F_{y_{1}}^{y_{2} \epsilon \epsilon^{\prime}}, F_{\epsilon^{\prime}}^{\epsilon y_{2} y_{1}}, F_{y_{2}}^{\epsilon \epsilon^{\prime} y_{1}}, F_{\epsilon}^{\epsilon^{\prime} y_{1} y_{2}}, F_{y_{1}}^{\epsilon^{\prime} \epsilon y_{2}}$

- $\left(\begin{array}{cc}-\frac{1}{\sqrt{2}} & -\frac{1}{\sqrt{2}} \\ \frac{1}{\sqrt{2}} & -\frac{1}{\sqrt{2}}\end{array}\right)$ for $F_{\epsilon}^{y_{1} y_{2} \epsilon^{\prime}}, F_{\epsilon^{\prime}}^{y_{2} y_{1} \epsilon}, F_{y_{1}}^{\epsilon \epsilon^{\prime} y_{2}}, F_{y_{2}}^{\epsilon^{\prime} \epsilon y_{1}}$

- $\left(\begin{array}{cc}\frac{1}{\sqrt{2}} & -\frac{1}{\sqrt{2}} \\ -\frac{1}{\sqrt{2}} & -\frac{1}{\sqrt{2}}\end{array}\right)$ for

$F_{\epsilon^{\prime}}^{y_{1} y_{2} \epsilon^{\prime}}, F_{y_{2}}^{y_{1} \epsilon^{\prime} \epsilon^{\prime}}, F_{\epsilon^{\prime}}^{y_{2} y_{1} \epsilon^{\prime}}, F_{y_{1}}^{y_{2} \epsilon^{\prime} \epsilon^{\prime}}, F_{\epsilon^{\prime}}^{\epsilon^{\prime} y_{1} y_{2}}, F_{\epsilon^{\prime}}^{\epsilon^{\prime} y_{2} y_{1}}, F_{y_{2}}^{\epsilon^{\prime} \epsilon^{\prime} y_{1}}, F_{y_{1}}^{\epsilon^{\prime} \epsilon^{\prime} y_{2}}$

- $\frac{1}{4}\left(\begin{array}{cc}-\frac{\sqrt{5}}{10} k^{2} & h \\ h & \frac{\sqrt{5}}{10} k^{2}\end{array}\right)$ for $F_{\epsilon}^{y_{1} \epsilon y_{1}}, F_{y_{1}}^{\epsilon y_{1} \epsilon}$

- $\frac{1}{4}\left(\begin{array}{cc}h & \frac{\sqrt{5}}{10} k^{2} \\ \frac{\sqrt{5}}{10} k^{2} & -h\end{array}\right)$ for $F_{\epsilon^{\prime}}^{y_{1} \epsilon y_{1}}, F_{\epsilon}^{y_{1} \epsilon^{\prime} y_{1}}, F_{y_{1}}^{\epsilon y_{1} \epsilon^{\prime}}, F_{y_{1}}^{\epsilon^{\prime} y_{1} \epsilon}$

- $\frac{1}{4}\left(\begin{array}{cc}\frac{\sqrt{5}}{10} h^{2} & k \\ k & -\frac{\sqrt{5}}{10} h^{2}\end{array}\right)$ for $F_{\epsilon}^{y_{1} \epsilon y_{2}}, F_{\epsilon}^{y_{2} \epsilon y_{1}}, F_{y_{2}}^{\epsilon y_{1} \epsilon}, F_{y_{1}}^{\epsilon y_{2} \epsilon}$

- $\frac{1}{4}\left(\begin{array}{cc}k & -\frac{\sqrt{5}}{10} h^{2} \\ -\frac{\sqrt{5}}{10} h^{2} & -\frac{\sqrt{5}}{20} h k^{2}\end{array}\right)$ for

$F_{\epsilon^{\prime}}^{y_{1} \epsilon y_{2}}, F_{\epsilon}^{y_{1} \epsilon^{\prime} y_{2}}, F_{\epsilon^{\prime}}^{y_{2} \epsilon y_{1}}, F_{\epsilon}^{y_{2} \epsilon^{\prime} y_{1}}, F_{y_{2}}^{\epsilon y_{1} \epsilon^{\prime}}, F_{y_{1}}^{\epsilon y_{2} \epsilon^{\prime}}, F_{y_{2}}^{\epsilon^{\prime} y_{1} \epsilon}, F_{y_{1}}^{\epsilon^{\prime} y_{2} \epsilon}$

- $\left(\begin{array}{cc}-\frac{1}{\sqrt{2}} & \frac{1}{\sqrt{2}} \\ -\frac{1}{\sqrt{2}} & -\frac{1}{\sqrt{2}}\end{array}\right)$ for $F_{y_{2}}^{y_{1} \epsilon \epsilon^{\prime}}, F_{y_{1}}^{y_{2} \epsilon^{\prime} \epsilon}, F_{\epsilon^{\prime}}^{\epsilon y_{1} y_{2}}, F_{\epsilon}^{\epsilon^{\prime} y_{2} y_{1}}$

- $\frac{1}{4}\left(\begin{array}{cc}\frac{\sqrt{5}}{10} k^{2} & -h \\ -h & -\frac{\sqrt{5}}{10} k^{2}\end{array}\right)$ for $F_{\epsilon^{\prime}}^{y_{1} \epsilon^{\prime} y_{1}}, F_{y_{1}}^{\epsilon^{\prime} y_{1} \epsilon^{\prime}}$

- $-\frac{\sqrt{5} h}{40}\left(\begin{array}{cc}h & \frac{k^{2}}{2} \\ \frac{k^{2}}{2} & -h\end{array}\right)$ for $F_{\epsilon^{\prime}}^{y_{1} \epsilon^{\prime} y_{2}}, F_{\epsilon^{\prime}}^{y_{2} \epsilon^{\prime} y_{1}}, F_{y_{2}}^{\epsilon^{\prime} y_{1} \epsilon^{\prime}}, F_{y_{1}}^{\epsilon^{\prime} y_{2} \epsilon^{\prime}}$

- $\frac{1}{4}\left(\begin{array}{cc}-\frac{\sqrt{5}}{10} k^{2} & -h \\ -h & \frac{\sqrt{5}}{10} k^{2}\end{array}\right)$ for $F_{\epsilon}^{y_{2} \epsilon y_{2}}, F_{y_{2}}^{\epsilon y_{2} \epsilon}$ 
- $\frac{1}{4}\left(\begin{array}{cc}-h & \frac{\sqrt{5}}{10} k^{2} \\ \frac{\sqrt{5}}{10} k^{2} & h\end{array}\right)$ for $F_{\epsilon^{\prime}}^{y_{2} \epsilon y_{2}}, F_{\epsilon}^{y_{2} \epsilon^{\prime} y_{2}}, F_{y_{2}}^{\epsilon y_{2} \epsilon^{\prime}}, F_{y_{2}}^{\epsilon^{\prime} y_{2} \epsilon}$

- $\frac{1}{4}\left(\begin{array}{cc}\frac{\sqrt{5}}{10} k^{2} & h \\ h & -\frac{\sqrt{5}}{10} k^{2}\end{array}\right)$ for $F_{\epsilon^{\prime}}^{y_{2} \epsilon^{\prime} y_{2}}, F_{y_{2}}^{\epsilon^{\prime} y_{2} \epsilon^{\prime}}$

- $\frac{\sqrt{5}}{10}\left(\begin{array}{cc}h & k \\ k & -h\end{array}\right)$ for $F_{\epsilon^{\prime}}^{\epsilon \epsilon \epsilon}, F_{\epsilon}^{\epsilon \epsilon \epsilon^{\prime}}, F_{\epsilon}^{\epsilon \epsilon^{\prime} \epsilon}, F_{\epsilon}^{\epsilon^{\prime} \epsilon \epsilon}$

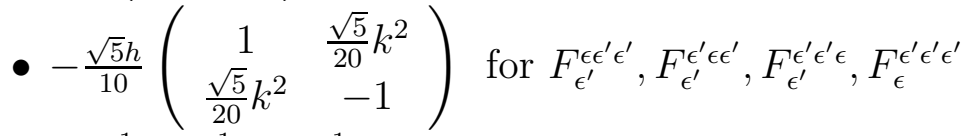

- $\left(\begin{array}{ccc}\frac{1}{2} & \frac{1}{2} & \frac{1}{\sqrt{2}} \\ \frac{1}{2} & \frac{1}{2} & -\frac{1}{\sqrt{2}} \\ \frac{1}{\sqrt{2}} & -\frac{1}{\sqrt{2}} & 0\end{array}\right)$ for $F_{y_{1}}^{y_{1} y_{1} y_{1}}, F_{y_{2}}^{y_{2} y_{2} y_{2}}$

- $\frac{1}{\sqrt{5}}\left(\begin{array}{ccc}1 & \sqrt{2} & \sqrt{2} \\ \sqrt{2} & -\frac{\sqrt{5}+1}{2} & \frac{\sqrt{5}-1}{2} \\ \sqrt{2} & \frac{\sqrt{5}-1}{2} & -\frac{\sqrt{5}+1}{2}\end{array}\right)$ for $F_{\epsilon}^{\epsilon \epsilon \epsilon}, F_{\epsilon^{\prime}}^{\epsilon^{\prime} \epsilon^{\prime} \epsilon^{\prime}}$

- $\frac{1}{\sqrt{5}}\left(\begin{array}{ccc}1 & -\sqrt{2} & -\sqrt{2} \\ \sqrt{2} & \frac{\sqrt{5}+1}{2} & -\frac{\sqrt{5}-1}{2} \\ \sqrt{2} & -\frac{\sqrt{5}-1}{2} & \frac{\sqrt{5}+1}{2}\end{array}\right)$ for $F_{\epsilon^{\prime}}^{\epsilon \epsilon \epsilon^{\prime}}, F_{\epsilon}^{\epsilon^{\prime} \epsilon^{\prime} \epsilon}$

- $\frac{1}{\sqrt{5}}\left(\begin{array}{ccc}-1 & \sqrt{2} & \sqrt{2} \\ \sqrt{2} & \frac{\sqrt{5}+1}{2} & -\frac{\sqrt{5}-1}{2} \\ \sqrt{2} & -\frac{\sqrt{5}-1}{2} & \frac{\sqrt{5}+1}{2}\end{array}\right)$ for $F_{\epsilon^{\prime}}^{\epsilon \epsilon^{\prime}}, F_{\epsilon}^{\epsilon^{\prime} \epsilon \epsilon^{\prime}}$

- $\frac{1}{\sqrt{5}}\left(\begin{array}{ccc}1 & \sqrt{2} & \sqrt{2} \\ -\sqrt{2} & \frac{\sqrt{5}+1}{2} & -\frac{\sqrt{5}-1}{2} \\ -\sqrt{2} & -\frac{\sqrt{5}-1}{2} & \frac{\sqrt{5}+1}{2}\end{array}\right)$ for $F_{\epsilon}^{\epsilon \epsilon^{\prime} \epsilon^{\prime}, F_{\epsilon^{\prime}}^{\epsilon^{\prime} \epsilon \epsilon}}$

B.1. $R$-symbols. We only list the $R$-symbols that are enough for our computational purpose. These data are from [13].

$$
\begin{aligned}
& R_{1}^{y_{1} y_{1}}=e^{\frac{6 \pi i}{5}}, R_{z}^{y_{1} y_{1}}=e^{\frac{\pi i}{5}}, R_{y_{2}}^{y_{1} y_{1}}=e^{\frac{4 \pi i}{5}} \\
& R_{1}^{\epsilon \epsilon}=-i, R_{y_{1}}^{\epsilon \epsilon}=e^{\frac{11 \pi i}{10}}, R_{y_{2}}^{\epsilon \epsilon}=e^{\frac{-\pi i}{10}}
\end{aligned}
$$

\section{Appendix C. Matrices of the generators of $\mathcal{B}_{4}$ IN A 1-QUPIT MODEL}

In this appendix, we show how to compute the matrices of the three generators $\sigma_{1}, \sigma_{2}, \sigma_{3}$ of $\mathcal{B}_{4}$ in a 1 -qupit model $V_{b}^{a a a a}$ with computational basis $\left\{\left|x_{i} y_{i}\right\rangle: 1 \leq i \leq p, p \geq 2\right\}$, where $a, b$ are two anyon types in a unitary modular category $\mathcal{C}$. The data we will use are some of the $F$-matrices and $R$-matrices, which are defined in Figure 7 and 8 , where 
UNIVERSAL QUANTUM COMPUTATION WITH METAPLECTIC ANYONS 21

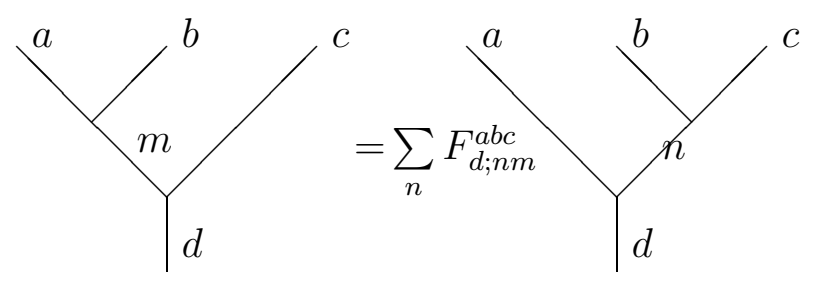

Figure 7. Definition of the $F$-matrix $F_{d ; n m}^{a b c}$

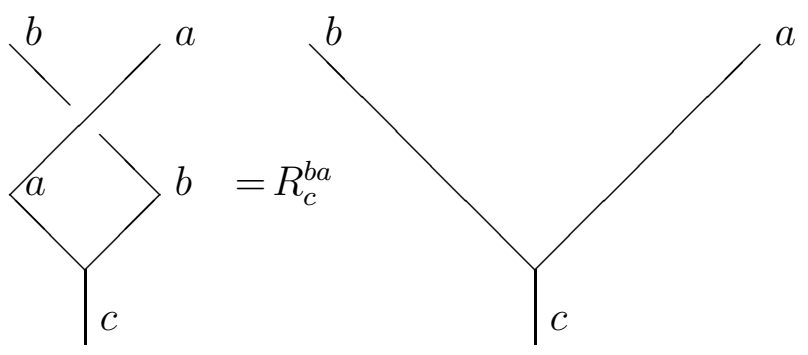

Figure 8. Definition of the $R$-matrix $R_{c}^{b a}$

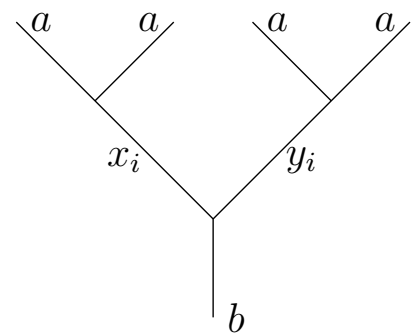

Figure 9. General 1-qupit model

$F_{d ; n m}^{a b c}$ is the $(n, m)$-entry of the matrix $F_{d}^{a b c}$, and $R_{c}^{b a}$ is a $1 \times 1 R$-matrix. For more detailed explanations, see Section 2 of [8], or [25].

See Figure 9 for the 1-qupit model $V_{b}^{a a a a}$. 

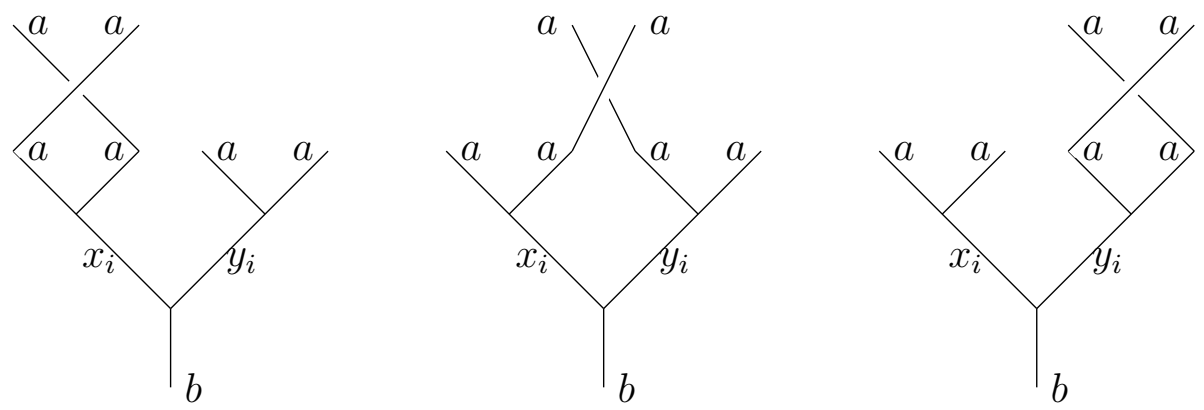

Figure 10. Braiding pictures for $\sigma_{1}, \sigma_{2}, \sigma_{3}$

Then the braiding pictures of $\sigma_{1}, \sigma_{2}, \sigma_{3}$ are given as shown in Figure 10. If we rewrite the braiding pictures in the computational basis, we get the matrices of the corresponding generators. Explicit illustrations are shown below.

By the definition of $R$-matrices in Figure 8 , the braiding picture for $\sigma_{1}$ can be written as that shown in Figure 11. Thus $\sigma_{1}$ is always the diagonal matrix with $(i, i)$-entry $R_{x_{i}}^{a a}$. Similarly, $\sigma_{3}$ is also a diagonal matrix with $(i, i)$-entry $R_{y_{i}}^{a a}$. The calculation of $\sigma_{2}$ is much more complicated as it involves change of bases using $F$-matrices. See figure 12 for the illustrations, where $F_{c ; d y_{i}}^{-1 a a a}$ is the $\left(d, y_{i}\right)$-entry of the inverse of the matrix $F_{c}^{a a a}$, and in the last picture of the equations, the pair $|f e\rangle$ could only be one of the basis elements in $\left\{\left|x_{j} y_{j}\right\rangle: 1 \leq j \leq p\right\}$. Let $|f e\rangle=\left|x_{j} y_{j}\right\rangle$, then the $(j, i)$-entry of $\sigma_{2}$ is given by the following:

$$
\left(\sigma_{2}\right)_{j, i}=\sum_{c, d} F_{b ; c x_{i}}^{a a y_{i}} F_{c ; d y_{i}}^{-1 a a a} R_{d}^{a a} F_{c ; y_{j} d}^{a a a} F_{b ; x_{j} c}^{-1 a a y_{j}}
$$

\section{REFERENCES}

[1] More powerful gates can be obtained by using higher genus surfaces and exotic boundary conditions. we will not use such protocols in this paper.

[2] Potentially, we could miss many intermediate complexity classes.

[3] Dorit Aharonov and Michael Ben-or. Fault-tolerant quantum computation with constant error rate. arXiv preprint quant-ph/9906129.

[4] Maissam Barkeshli and Xiao-Gang Wen. Anyon condensation and continuous topological phase transitions in non-abelian fractional quantum hall states. Physical review letters, 105(21):216804, 2010.

[5] P Oscar Boykin, Tal Mor, Matthew Pulver, Vwani Roychowdhury, and Farrokh Vatan. On universal and fault-tolerant quantum computing: a novel basis and a new constructive proof of universality for Shor's basis. In Foundations of 
UNIVERSAL QUANTUM COMPUTATION WITH METAPLECTIC ANYONS 23

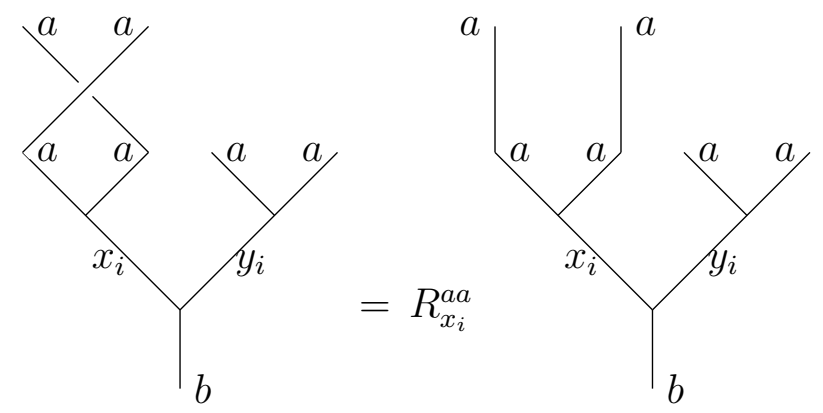

Figure 11. Calculating $\sigma_{1}$

Computer Science, 1999. 40th Annual Symposium on, pages 486-494. IEEE, 1999.

[6] Jean-Luc Brylinski and Ranee Brylinski. Universal quantum gates. Mathematics of Quantum Computation, 79, 2002.

[7] David J Clarke, Jason Alicea, and Kirill Shtengel. Exotic non-abelian anyons from conventional fractional quantum hall states. Nature communications, 4:1348, 2013.

[8] Shawn X Cui, Seung-Moon Hong, and Zhenghan Wang. Universal quantum computation with weakly integral anyons. arXiv preprint arXiv:1401.7096, 2014.

[9] WM Fairbairn, T Fulton, and WH Klink. Finite and disconnected subgroups of $S U(3)$ and their application to the elementary-particle spectrum. Journal of mathematical physics, 5(8):1038-1051, 1964.

[10] Michael H Freedman, Michael Larsen, and Zhenghan Wang. A modular functor which is universal for quantum computation. Commun. Math. Phys, 227:605$622,2002$.

[11] Michael H Freedman and Claire I Levaillant. Interferometry versus projective measurement of anyons. arXiv preprint arXiv:1501.01339, 2015.

[12] Matthew B. Hastings, Chetan Nayak, and Zhenghan Wang. Metaplectic anyons, majorana zero modes, and their computational power. Physical Review B, 87(16):165421, 2013.

[13] Matthew B. Hastings, Chetan Nayak, and Zhenghan Wang. On metaplectic modular categories and their applications. Communications in Mathematical Physics, pages 1-24, 2014.

[14] Camille Jordan. Mmoire sur les quations diffrentielles linaires intgrale algbrique. Journal fr die reine und angewandte Mathematik, 84:89-215, 1877.

[15] A Yu Kitaev. Quantum computation, ph/cs 219, winter 2007, problem set 3. http://www.theory.caltech.edu/ preskill/ph219/prob7_07-kitaev.pdf

[16] A Yu Kitaev. Quantum computations: algorithms and error correction. Russian Mathematical Surveys, 52(6):1191-1249, 1997.

[17] C. Levaillant. From ancilla to quantum gate. arXiv preprint arXiv:1501.02841, 2015.

[18] G Miller. Theory and Applications of Finite Groups. Applewood Books, 2012. 

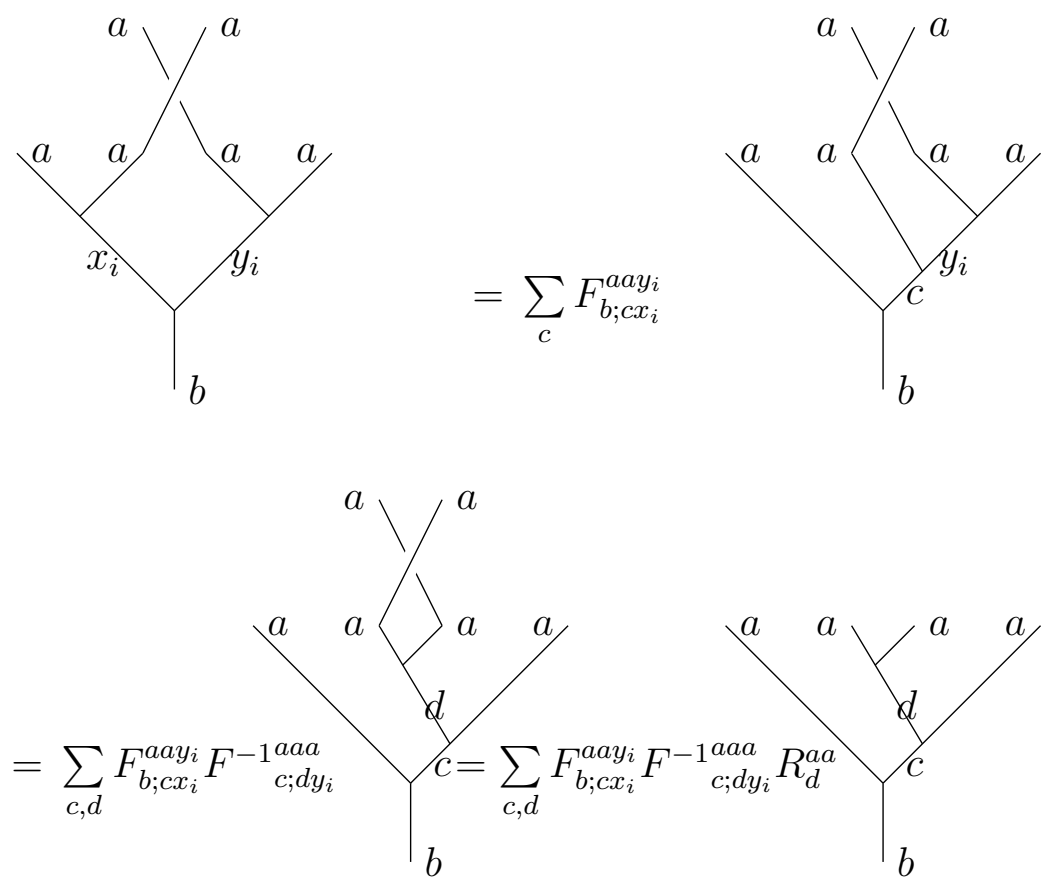

$=\sum_{c, d, e} F_{b ; c x_{i}}^{a a y_{i}} F_{c ; d y_{i}}^{-1} R_{d}^{a a a} F_{c ; e d}^{a a a}$

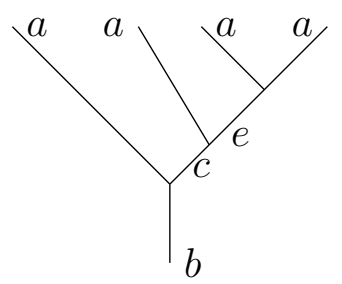

$=\sum_{c, d, e, f} F_{b ; c x_{i}}^{a a y_{i}} F_{c ; d y_{i}}^{-1} R_{d}^{a a a} F_{c ; e d}^{a a a} F_{b ; f c}^{-1} a a e$

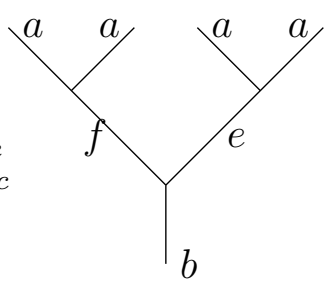

Figure 12. Calculating $\sigma_{2}$

[19] Deepak Naidu and Eric C Rowell. A finiteness property for braided fusion categories. Algebras and representation theory, 14(5):837-855, 2011.

[20] Michael A Nielsen and Isaac L Chuang. Quantum computation and quantum information. Cambridge university press, 2010. 
UNIVERSAL QUANTUM COMPUTATION WITH METAPLECTIC ANYONS 25

[21] Michael R Peterson, Yang-Le Wu, Meng Cheng, Maissam Barkeshli, Zhenghan Wang, and Sankar Das Sarma. Abelian and non-abelian states in $\nu=2 / 3$ bilayer fractional quantum hall systems. arXiv preprint arXiv:1502.02671, 2015.

[22] N Read and E Rezayi. Beyond paired quantum hall states: parafermions and incompressible states in the first excited landau level. Physical Review $B, 59(12): 8084,1999$.

[23] Eric C Rowell and Hans Wenzl. $S O(N) \_2$ braid group representations are Gaussian. arXiv preprint arXiv:1401.5329, 2014.

[24] Geoffrey C Shephard and John A Todd. Finite unitary reflection groups. Canad. J. Math, 6(2):274-301, 1954.

[25] Zhenghan Wang. Topological quantum computation, volume 112. Amer Mathematical Society, 2010.

${ }^{1}$ Department of Mathematics, University of California, Santa BarBARA, CA 93106

E-mail address: xingshan@math.ucsb.edu, zhenghwa@math.ucsb.edu

${ }^{2}$ Microsoft Research, Station Q, University of California, Santa BARBARA, CA 93106

E-mail address: zhenghwa@microsoft.com 